\title{
Seltene Krankheiten sind leider nicht selten
}

\section{Manuela Stiera, Agnes Genewein ${ }^{b}$}

${ }^{a}$ Dipl. PR-Beraterin, Initiantin und Geschäftsleiterin Kinder mit seltenen Krankheiten - Gemeinnütziger Förderverein; ${ }^{b}$ Dr. med., EMBA HSG, MAE, Co-Leiterin des Netzwerks Rare Diseases Nordwest und Zentralschweiz, Neonatologin am Universitäts-Kinderspital beider Basel

\begin{abstract}
Beinahe fünf Prozent der in der Schweiz lebenden Kinder und Jugendlichen sind von einer seltenen Krankheit betroffen. Aufgrund der rund 8000 verschiedenen seltenen Krankheiten ist es für Ärzte besonders schwierig, Diagnosen und Prognosen stellen zu können. Was entsteht, ist eine Situation der Ungewissheit für alle Beteiligten. Der Förderverein für Kinder mit seltenen Krankheiten möchte hier helfen.
\end{abstract}

Was selten ist, wird häufig kaum beachtet. Das ist bei seltenen Krankheiten nicht anders, wobei hier der Begriff trügerisch ist, denn bei einer Prävalenz von weniger als einer von 2000 Personen ist zwar die einzelne Krankheit selten, nicht aber die Gesamtzahl der Betroffenen. So sind alleine in der Schweiz rund 500000 Menschen von den Folgen einer seltenen Krankheit betroffen. Rund 75\% der seltenen Krankheiten werden im Kindesalter entdeckt. Die Krankheiten wirken sich dabei nicht nur auf die betroffenen Kinder aus, sondern auch auf ihre Familie, die berufliche Situation der Eltern und ihr Umfeld. Arzt-, Spital- und Therapiebesuche sind oft an der Tagesordnung, und viele der Betroffenen benötigen lebenslang Unterstützung.

\section{Ein Förderverein für betroffene Familien}

Im Februar 2014 habe ich mich deshalb dazu entschieden, den gemeinnützigen Förderverein für Kinder mit seltenen Krankheiten zu gründen. Gezielt wählte ich damals die Vorstandsmitglieder aus, um breit abgestützt zu sein. Mit Prof. Dr. med. Thierry Carrel, Direktor der Universitätsklinik für Herz- und Gefässchirurgie am Inselspital Bern, als Präsidenten und Prof. Dr. med. Matthias Baumgartner, Leiter der Abteilung für seltene Stoffwechselkrankheiten am Kinderspital Zürich, sind ausgewiesene Fachpersonen im Bereich der seltenen Krankheit mit an Bord, die sich aktiv für die betroffenen Familien einsetzen. Um auch deren Perspektive gebührend zu vertreten, ist ein betroffener Vater Teil des Vorstands.

Der Förderverein für Kinder mit seltenen Krankheiten hat sich drei Ziele gesetzt: Er will die betroffenen Fami-

\section{Résumé}

Ce qui est rare ne suscite souvent que peu d'attention. II en va ainsi des maladies rares, bien que le terme soit ici trompeur. Presque 5\% des enfants et des jeunes qui vivent en Suisse sont en effet atteints d'une telle maladie. Celles-ci ne touchent pas seulement les enfants eux-mêmes, mais aussi leur famille, la situation professionnelle des parents et leur environnement. Certes, les très vastes connaissances acquises grâce aux plus récentes techniques d'analyse génétique autorisent aujourd'hui des diagnostics et pronostics toujours plus précis. Mais en dépit de ces progrès, les visites chez le médecin, le thérapeute ou à l'hôpital restent à l'ordre du jour des familles concernées. Nombreux sont ainsi les enfants et les jeunes qui nécessiteront d'être soutenus à vie. La Förderverein für Kinder mit seltenen Krankheiten (Association de soutien pour les enfants atteints de maladies rares) poursuit donc trois objectifs: mettre les familles concernées en relation les unes avec les autres pour leur montrer qu'elles ne sont pas seules face à leur sort. Organiser des aides financières directes et les mettre rapidement à disposition, durablement et sans bureaucratie superflue. Sensibiliser le public, et présenter ce qu'accomplissent les familles concernées par les maladies rares.

lien miteinander vernetzen, um ihnen zu zeigen, dass sie mit ihrem Schicksal nicht alleine sind. Der Verein organisiert finanzielle Direkthilfe und stellt diese schnell, unbürokratisch zur Verfügung. Zudem sensi- 


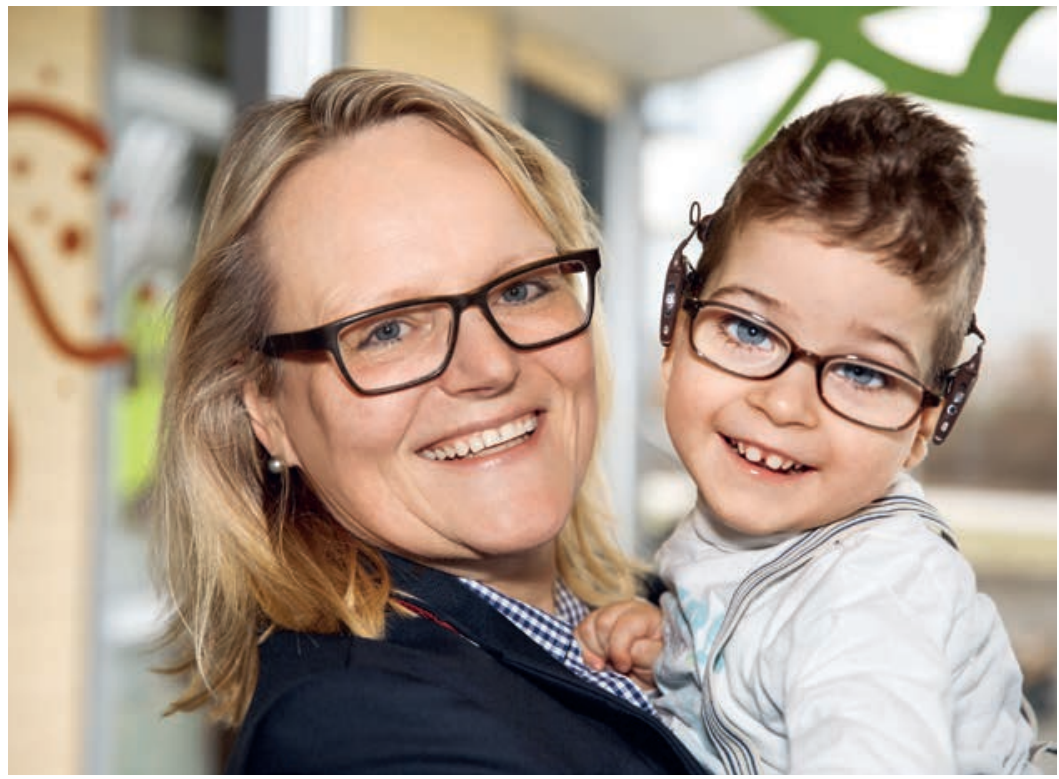

Manuela Stier und Rayan, der von einer seltenen Krankheit betroffen ist.

bilisiert der Förderverein die Öffentlichkeit und zeigt, was es bedeutet, betroffen zu sein. Im Folgenden erläutern wir, weshalb diese drei Ziele für die betroffenen Familien so wichtig sind.

\section{Umgang mit der Ungewissheit}

Für die Familien gleicht das Leben mit einer seltenen Krankheit einer Achterbahnfahrt der Gefühle. Oftmals beginnt die Fahrt damit, dass ihr Kind krank ist, aber niemand weiss im ersten Moment, woran das Kind leidet. Aufgrund der rund 8000 verschiedenen seltenen Krankheiten ist es für Ärzte schwierig, eine Diagnose zu stellen. Oftmals sind dazu aufwendige Genuntersuchungen notwendig, deren Kostengutsprache meist in einem finanziellen Kampf mit der Versicherung endet. Die Familien durchleben hier eine Zeit der Ungewissheit und Hilflosigkeit. Haben sie keine Diagnose, gibt es folglich auch keine Prognose zur Lebenserwartung oder zum weiteren Krankheitsverlauf. Besonders schlimm wird es für betroffene Familien dann, wenn sie sich nicht ernst genommen fühlen. Nicht selten erleben wir, dass uns Familien davon berichten, dass sie erstmals als übervorsichtige Eltern abgestempelt werden oder dass das Kind «bloss» entwicklungsverzögert sei. Auch hören wir oft von überforderten Ärzten oder davon, dass das Kind von einer Stelle zur nächsten weitergereicht wird, da niemand die Verantwortung übernehmen will. Die betroffenen Familien machen den Ärzten keine Vorwürfe, schliesslich sind auch diese nicht täglich mit seltenen Krankheiten konfrontiert. Sie wünschen sich aber, dass man ihnen zuhört und ihre Probleme ernst nimmt. Denn als sensible Eltern kennen sie ihr Kind bestens, und oftmals haben sie sich zu wahren Experten rund um die Symptome und möglichen Krankheitsbilder ihrer Kinder entwickelt.

Unser Förderverein will hier helfen. Einerseits indem wir die betroffenen Familien miteinander vernetzen. Sei dies über unsere geschlossene Selbsthilfegruppe auf Facebook mit über 210 vernetzten Eltern oder an unseren Events, die stets viel Zeit zum gemeinsamen Austausch bieten. Rund 1000 Personen nutzen jedes Jahr unsere kostenlosen Events zum gegenseitigen Austausch, und über 120 freiwillige Helferinnen und Helfer sind vor Ort, um den Betroffenen eine kurze Auszeit vom Alltag zu bieten. Dabei ist es wichtig, zu verstehen, dass viele Herausforderungen der betroffenen Familien unabhängig vom eigentlichen Krankheitsbild sind und es ihnen hilft, Menschen mit ähnlichem Schicksal zu treffen. Andererseits aber möchten wir auch die Ärzte und medizinische Fachpersonen mit gezielter Öffentlichkeitsarbeit für das wichtige Thema «seltene Krankheiten» sensibilisieren. Dies tun wir etwa mit unserem ersten KMSK-Wissensbuch Seltene Krankheiten - Einblicke in das Leben betroffener Familien. Darin berichten 17 betroffene Familien von ihrem Alltag und ihrem Schicksal - von der Diagnosestellung über den Familienalltag bis hin zum Tod. Die behandelnden Ärzte, Therapeuten und Genetiker teilen in Interviews ihre Erfahrungen. Zudem versuchen wir 2019, mit neuen Wissenstalks «Hören» bei Sonova und «Robotik» in der Rehaklinik Zürich Affoltern gezielte Informationsarbeit bei Familien und Ärzten zu leisten.

\section{Bedeutung der seltenen Krankheiten nimmt zu}

In den vergangenen vier Jahren konnten wir einen Wandel in der Wahrnehmung der seltenen Krankheiten beobachten. In der Pharmabranche etwa werden seltene Krankheiten heute nicht mehr einfach als unrentabel abgestempelt. Das Thema wird auf vielfältige Weise aufgegriffen, sei es mittels Studien mit Betroffenen, Forschung an neuen Medikamenten oder mittels sozialen Engagements zugunsten der betroffenen Familien. Kinderspitäler wie das Universitäts-Kinderspital beider Basel informieren ihre Patienten und die Öffentlichkeit aktiv über seltene Krankheiten. Ein sinnvoller Schritt, wenn man bedenkt, dass heute jeder dritte Patient einer universitären Kinderklinik von einer seltenen Krankheit betroffen ist. Und auch auf politischer Ebene wurde mit dem «Konzept Seltene Krankheiten» des Bundes ein erster Lösungsvorschlag 
zur Problematik ausgearbeitet. So sollen etwa drei Referenzzentren künftig für eine bessere Diagnose und eine gezieltere Behandlung sorgen. Ein Schritt, der aus Sicht der betroffenen Familien gemischte Gefühle auslöst. Einerseits sind solche Referenzzentren mit teilweise langen Anreisewegen verbunden, was besonders für schwerbehinderte Patienten einen grossen Aufwand darstellt. Auf der anderen Seite aber wünschen sich die Familien auch eine zentrale Anlaufstelle. Das Wichtigste für die betroffenen Familien ist aber, dass die Verbesserungsmassnahmen schnell umgesetzt werden. Denn morgen könnte es leider schon zu spät sein.

\section{Der lange Weg bis zur Diagnose}

Symptome sind die Puzzleteile, die zu einer Diagnose führen. Passen die Symptome nicht in ein klassisches Schema einer Krankheit, beginnt die detektivische Arbeit: Einbezug anderer Kollegen mit einer spezifischen Expertise national und international, ausgedehnte Literaturrecherche in internationalen medizinischen $\mathrm{Da}$ tenbanken. Daraus erfolgen oft weitere Untersuchungen, weitere Recherchen, eine weitere Annäherung an die Diagnose. Diese detektivischen Schlaufen engen zunehmend die in Frage kommenden Krankheiten ein, bis sich das behandelnde Team im besten Fall auf eine Diagnose festlegen kann. Das sind oft lange Wege, die personal- und zeitintensiv sind.

Erst mit einer gesicherten Diagnose kann die richtige Therapie eingeleitet werden. Und oft ermöglicht erst das Ansprechen auf eine Therapie eine Vorhersage über den Verlauf der Krankheit. Das aber ist für die Patienten und die Familien entscheidend. Erst dann können sie sich darauf einstellen und ihr Leben darum herum organisieren.

Heute werden dank neuester genetischer Analysetechniken umfangreichere Erkenntnisse auf genetischer Ebene erzielt. Dies führt in der Regel zu exakteren Diagnosen und Prognosen. Dank der raschen Weiterentwicklung in der Genetik entstehen heute aber auch schwierig zu interpretierende Resultate und für die Betroffenen wieder Ungewissheit. Diese Herausforderungen werden durch weitere Forschung und wissenschaftliche Erkenntnisse geringer werden. Wenn es schliesslich gelingt, mit Hilfe der genetischen Diagnose eine entsprechende Therapie einzuleiten, dann besteht Hoffnung für die Patienten.

\section{Finanzielle Direkthilfe}

Die medizinischen Probleme alleine sind es aber nicht, die das Leben der betroffenen Familien zu einem Ge-

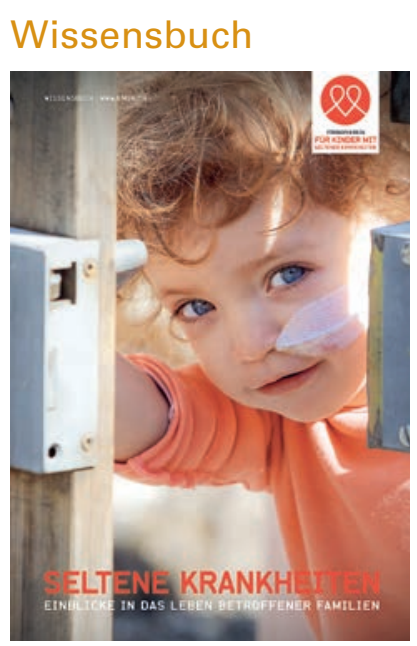

"Seltene Krankheiten Einblicke in das Leben betroffener Familien»

Bestellen Sie kostenlos dieses informative Werk für Sie, Ihre Kollegen und Patienten bei manuela.stier[at]kmsk.ch

fühlschaos machen. Oftmals stehen die Familien auch vor finanziellen Problemen. Natürlich werden die meisten medizinischen Leistungen und Hilfsmittel von der Krankenkasse oder der IV bezahlt. Allerdings ist dies oft mit einem grossen bürokratischen und teilweise gar juristischen Aufwand verbunden. Doch es gibt auch immer wieder Situationen, in denen eine finanzielle Unterstützung fehlt. So etwa für erfolgversprechende alternative Therapien, für eine bessere Mobilität oder für Auszeiten für die pflegenden Familienmitglieder. Hier schliesst der Förderverein die Lücken, die weder durch die IV oder die Krankenkasse noch durch die Familien selbst finanziert werden können.

\section{Lebensqualität schenken}

Kinder sind das höchste Gut, das wir haben. Entsprechend ist es unsere Aufgabe, sie zu schützen und ihnen beim Erwachsenwerden zu helfen. Sind die Kinder dann noch von einer seltenen Krankheit betroffen, gilt dieses Gebot umso mehr. Um den betroffenen Familien auf medizinischer, gesellschaftlicher und finanzieller Ebene helfen zu können, ist der gemeinsame Dialog aller Beteiligten die wichtigste Grundvoraussetzung. Erst das gegenseitige Verständnis führt nämlich dazu, dass wir gemeinsam die Lebenssituation der betroffenen Familien verbessern können. Und dafür setzen wir uns ein - hoffentlich auch mit Ihnen!

Bildnachweis

Foto: Martina Ronner-Kammer 